\title{
A RUNGE THEOREM FOR HARMONIC FUNCTIONS ON CLOSED SUBSETS OF RIEMANN SURFACES
}

\author{
THOMAS BAGBY
}

(Communicated by Irwin Kra)

\begin{abstract}
Let $F$ be a closed subset of a Riemann surface $\Omega$. It is shown that every function which is harmonic on a neighborhood of $F$ can be uniformly approximated on $F$ by functions which are harmonic on $\Omega$ except for poles.
\end{abstract}

1. Introduction. In this paper we prove a theorem of Runge type for uniform approximation by harmonic functions on closed subsets of Riemann surfaces. If $\Omega$ is a Riemann surface, a continuous function $h: \Omega \rightarrow[-\infty, \infty]$ will be said to be essentially harmonic provided that it is harmonic except for logarithmic singularities; that is, provided that for each point $p \in \Omega$ there exist a local coordinate map $z$ near $p$, a real number $c$, and a harmonic function $w$ near $p$ such that $h(q) \equiv w(q)+c \log |z(q)-z(p)|$ in some neighborhood of $p$.

THEOREM. Let $F$ be a closed subset of a Riemann surface $\Omega$, and let $f$ be a harmonic function on an open set containing $F$. If $\varepsilon>0$, then there exists an essentially harmonic function $h$ on $\Omega$ such that $\sup _{F}|f-h|<\varepsilon$.

The analogous assertion for holomorphic functions would be false. In fact, from work of Gauthier and Hengartner [4] one sees that there exists a Riemann surface $\Omega$, a closed set $F \subset \Omega$, and a holomorphic function $f$ on a neighborhood of $F$, with the following property: if $h$ is holomorphic on $\Omega$ except for isolated singularities, then $\sup _{F}|f-h|>1$. This was pointed out to the author by Gauthier.

Special cases of the theorem follow from work of Lax, Malgrange, Pfluger, Boivin, Gauthier, Goldstein and Ow; for a discussion of this work we refer to [5, Chapitre 2] and [2]. In particular, we note that the theorem is proved in [5, Corollaire 2.3.9] under the additional assumption that $F$ is "essentially of finite genus" (for terminology see [5, p. 29]); and the theorem is proved in [5, Théorème 2.3.5] if "essentially harmonic" is replaced by "harmonic on the complement of a discrete subset of $\Omega$." In a forthcoming joint paper with P. M. Gauthier the results proved here will be combined with arguments concerning balayage of poles; in particular, we will show that under appropriate additional hypotheses one can obtain approximating functions $h$ which are harmonic on all of $\Omega$.

We recall some well known results concerning Riemann surfaces in $\S 2$. The proof of the theorem is then given in $\S 3$.

2. Notation and preliminaries. We set $B_{r}=\{z \in \mathbf{C}:|z|<r\}$. The notation $f: A \rightarrow B$ indicates that the function $f: A \rightarrow B$ is onto.

Received by the editors November 21, 1985 and, in revised form, January 6, 1987.

1980 Mathematics Subject Classification (1985 Revision). Primary 30F 15; Secondary 30E10. 
A Riemann surface is a connected complex-analytic manifold of complex dimension one. If $\Omega$ is a Riemann surface, a 2-form $\alpha$ on $\Omega$ assigns a function $\alpha_{z}$ for each coordinate map $z=x+i y$, such that on overlapping coordinate regions we have $\alpha_{\varsigma}=\alpha_{z}|d z / d \zeta|^{2}$; we write $\alpha=\alpha_{z} d x \wedge d y=i \alpha_{z} d z \wedge d \bar{z} / 2$. If $\alpha$ is a continuous 2 -form, corresponding to a function $\alpha_{z}$ for each local coordinate $z$, then $|\alpha|$ denotes the continuous 2-form which assigns the function $\left|\alpha_{z}\right|$ to each local coordinate $z$.

Every Riemann surface is either hyperbolic or parabolic (see [6]). In the remainder of this section we summarize some facts associated with this classification.

If $\Omega$ is a hyperbolic Riemann surface, then there exists a Green's kernel for $\Omega$, which is the unique mapping $q: \Omega \times \Omega \rightarrow(0, \infty]$ with the following properties [6, Chapter V, $\S 2,8]$.

(G1) For each point $q \in \Omega$, the function $\Omega \backslash\{q\} \ni p \rightarrow g(p, q)$ is harmonic.

(G2) If $\omega$ is a parametric disc for $\Omega$, and $z: \omega \rightarrow B_{1}$ is a conformal homeomorphism, then we may write $g(p, q)=-\log |z(p)-z(q)|+v(p, q)$ for $p, q \in \omega$, where $v: \omega \times \omega \rightarrow \mathbf{R}$ is (jointly) continuous, and, for each point $a \in \omega$, the functions $\omega \ni p \rightarrow v(p, a)$ and $\omega \ni q \rightarrow v(a, q)$ are harmonic.

(G3) If $q \in \Omega$, and $u$ is any bounded harmonic function on $\Omega$ with $g(\cdot, q) \geq u \geq 0$, then $u \equiv 0$.

(G4) $g(p, q)=g(q, p)$ for all points $p, q \in \Omega$.

If $\Omega$ is an open parabolic Riemann surface, there is an Evans kernel for $\Omega$, which is a function $e: \Omega \times \Omega \rightarrow(-\infty, \infty]$ with the following properties [6, Chapter $\mathrm{V}, \S 3$, 13] (these properties are analogous to those for $e(p, q) \equiv-\log |p-q|$ when $\Omega=\mathbf{C}$ ):

(E1) for each point $q \in \Omega$, the function $\Omega \backslash\{q\} \ni p \rightarrow e(p, q)$ is harmonic.

(E2) if $\omega$ is a regular subregion of $\Omega$, we may write $e(p, q)=g_{\omega}(p, q)+v(p, q)$ for $p, q \in \omega$, where $g_{\omega}$ is the Green's function for $\omega$, the function $v: \omega \times \omega \rightarrow \mathbf{R}$ is (jointly) continuous, and, for each point $a \in \omega$, the functions $\omega \ni p \rightarrow v(p, a)$ and $\omega \ni q \rightarrow v(a, q)$ are harmonic.

(E3) for each point $q \in \Omega$, there is a compact set $K \subset \Omega$ such that $e(p, q) \leq 0$ for $p \in \Omega \backslash K$.

(E4) if $q \in \Omega$ and $q^{\prime} \in \Omega$, then there is a compact set $K=K\left(q, q^{\prime}\right)$ such that the function $\Omega \backslash K \ni p \rightarrow e\left(p, q^{\prime}\right)-e(p, q)$ is bounded.

(E5) $e(p, q)=e(q, p)$ for all points $p, q \in \Omega$.

We will need the following maximum principle for parabolic Riemann surfaces: if $\Omega$ is an open parabolic Riemann surface, $\omega \subset \subset \Omega$ is a regular subregion, and $u: \Omega \backslash \omega \rightarrow \mathbf{R}$ is a bounded continuous function which is harmonic on $\Omega \backslash \bar{\omega}$ and is $\leq 0$ on $\partial \omega$, then $u \leq 0$ on $\Omega \backslash \omega$. (See $[6$, Chapter V, $\S 2,7 \mathrm{E}]$.)

3. Proof of the theorem. If $\Omega$ is compact, then $F$ is compact, and the theorem is well known [5, Théorème 2.1.8].

For the rest of the paper we assume that $\Omega$ is an open Riemann surface. We let $E: \Omega \times \Omega \rightarrow(-\infty, \infty)$ denote the Green's kernel if $\Omega$ is hyperbolic, and the Evans kernel if $\Omega$ is parabolic. The operator $\partial \bar{\partial}$ defines a mapping from $C^{\infty}$ functions on $\Omega$ to 2 -forms on $\Omega$; the singularity of $E(\cdot, q)$ at $q$ has been chosen so that $E(\cdot, q) / \pi i$ may be used as a type of fundamental solution for this operator, as stated in the following lemma. 
LEMMA 1. Let $\alpha$ be a smooth 2-form on $\Omega$ having compact support. Then the function

$$
u(p)=\frac{1}{\pi i} \int E(p, q) \alpha(q), \quad p \in \Omega,
$$

is smooth and satisfies $\partial \bar{\partial} u=\alpha$.

Since results of this type are well known, we will only outline the proof. By use of a partition-of-unity, the proof may be reduced to the case where the support of $\alpha$ is a compact subset of a parametric disk $\omega \subset \subset \Omega$. In this case the desired properties of $u$ on $\omega$ follow from the fact that $(2 / \pi) \log |z|$ is a fundamental solution for the operator $\partial^{2} / \partial z \partial \bar{z}$ in $\mathbf{C}$; and the desired properties of $u$ in a neighborhood of $\Omega \backslash \omega$ follow from the fact that for each point $q \in \Omega$ the function $E(\cdot, q)$ is harmonic on $\Omega \backslash\{q\}$.

LEMMA 2. Let $D$ be an open subset of $\Omega$, and $q \in D$. If $\varepsilon>0$, then there exists a parametric disc $N$ such that $q \in N \subset \subset D$ and $\left|E(p, q)-E\left(p, q_{1}\right)\right| \leq \varepsilon$ for $p \in \Omega \backslash D, q_{1} \in N$.

PROOF. We may assume without loss of generality that there is an open set $\omega$ with $D \subset \subset \omega \subset \subset \Omega$ and a conformal homeomorphism $h: \omega \rightarrow B_{1}$ such that $h(q)=0$ and the image of $D$ is $B_{\rho}, \rho<1$. Since the mapping $\partial B_{\rho} \times \overline{B_{\rho / 2}} \ni$ $(z, \varsigma) \rightarrow E\left(h^{-1}(z), h^{-1}(\varsigma)\right)$ is uniformly continuous, we may select a parametric disc $N$ such that $q \in N \subset \subset D$ and

$$
\left|E(p, q)-E\left(p, q_{1}\right)\right| \leq \varepsilon / 3 \text { for } p \in \partial D, q_{1} \in \bar{N} .
$$

To complete the proof of the lemma we consider separately the case where $\Omega$ is parabolic and the case where $\Omega$ is hyperbolic.

Suppose first that $\Omega$ is parabolic, and let $q_{1} \in N$ be fixed. It follows easily from property $\mathrm{E} 4$ in $\S 2$ that the function $\Omega \backslash D \ni p \rightarrow E(p, q)-E\left(p, q_{1}\right)$ is bounded. From (1) and the maximum principle for parabolic surfaces we conclude that $\mid E(p, q)-$ $E\left(p, q_{1}\right) \mid \leq \varepsilon / 3<\varepsilon$ for all $p \in \Omega \backslash D$. This proves Lemma 2 in case $\Omega$ is parabolic.

For the rest of the proof of Lemma 2 we assume that $\Omega$ is hyperbolic. Let $D \subset \subset$ $\Omega_{1} \subset \subset \Omega_{2} \subset \subset \cdots$, where each $\Omega_{j}$ is a regular subregion of $\Omega$ and $\Omega=\bigcup_{j=1}^{\infty} \Omega_{j}$. Let $g_{j}$ be the Green's function for $\Omega_{j}$; from $[6$, Chapter $\mathrm{V}, \S 2,8 \mathrm{~A}]$ it follows that

$$
g_{j} \uparrow E .
$$

(In the proof that follows we will combine (2) with the maximum principle for regular subregions, a technique used in [5, pp. 72-73].) From (2) and Dini's theorem we conclude that $g_{j} \uparrow E$ uniformly (as real-valued functions) on the compact set $(\partial D) \times \bar{N}$, since the limit $E$ is real-valued and continuous there. We thus see that there exists an index $J$ such that for $p \in \partial D$ and $j \geq J$ we have

$$
\left|g_{j}\left(p, q_{1}\right)-E\left(p, q_{1}\right)\right| \leq \varepsilon / 3, \quad q_{1} \in \bar{N},
$$

and hence we have

$$
\begin{aligned}
\left|g_{j}(p, q)-g_{j}\left(p, q_{1}\right)\right| \leq & \left|g_{j}(p, q)-E(p, q)\right|+\left|E(p, q)-E\left(p, q_{1}\right)\right| \\
& +\left|E\left(p, q_{1}\right)-g_{j}\left(p, q_{1}\right)\right| \\
\leq & \varepsilon / 3+\varepsilon / 3+\varepsilon / 3=\varepsilon, \quad q_{1} \in \bar{N} .
\end{aligned}
$$


We now claim that for each fixed integer $j \geq J$ we have

$$
\left|g_{j}(p, q)-g_{j}\left(p, q_{1}\right)\right| \leq \varepsilon \quad \text { if } p \in \Omega_{j} \backslash D \text { and } q_{1} \in N .
$$

To prove this claim, we fix $q_{1} \in N$, and consider the function

$$
G(p) \equiv g_{j}(p, q)-g_{j}\left(p, q_{1}\right), \quad p \in \overline{\Omega_{j}} \backslash D .
$$

We have $G \equiv 0$ on $\partial \Omega_{j}$, and from (3) we see that $|G| \leq \varepsilon$ on $\partial D$; from the usual maximum principle for harmonic functions it follows that $|G| \leq \varepsilon$ on $\overline{\Omega_{j}} \backslash D$, which proves the claim.

The lemma now follows by taking fixed points $p \in \Omega \backslash D$ and $q_{1} \in N$, and letting $j \rightarrow \infty$ in the claim.

We now complete the proof of the theorem stated in $\S 1$. The proof makes use of the Mittag-Leffler technique, and should be compared with the proof of [2, Théorème 1]. Let $N \supset F$ be open, and $f: N \rightarrow \mathbf{R}$ be harmonic. There exists a function $\chi \in C^{\infty}(\Omega)$ such that $\chi \equiv 1$ on an open neighborhood of $F$, and $\chi \equiv 0$ on an open neighborhood of $\Omega \backslash N[\mathbf{7}, 2.2 .14]$. Let $u=\chi f$, with the understanding that $u \equiv 0$ on $\Omega \backslash N$. Let $\beta$ be the $C^{\infty} 2$-form $\partial \bar{\partial} u$, so that $\operatorname{supp} \beta$ is a closed subset of $\Omega$ which does not intersect $F$.

It is possible to find a sequence $\left\{K_{j}\right\}_{j=1}^{\infty}$ of compact subsets of $\Omega$ such that $\Omega=\bigcup_{j=1}^{\infty} K_{j}$ and, for each integer $j \geq 1, \Omega \backslash K_{j}$ has no relatively compact connected components and $K_{j} \subset$ int $K_{j+1}$; this is easily proved using [7,3.10.3] and the fact that a Riemann surface has a second countable topology. For each $j \geq 1$ we let $\psi_{j} \in C_{0}^{\infty}$ (int $K_{j+1}$ ) be identically equal to one on a neighborhood of $K_{j}$. We define $\Omega_{1}=\operatorname{int} K_{2}, \Omega_{j}=\left(\right.$ int $\left.K_{j+1}\right) \backslash K_{j-1}$ for $j \geq 2, \varphi_{1}=\psi_{1}$, and $\varphi_{j}=\psi_{j}-\psi_{j-1}$ for $j \geq 2$. Then $\operatorname{supp} \varphi_{j} \subset \Omega_{j}$ for $j \geq 2$, and $\sum_{j=1}^{k} \varphi_{j} \equiv \psi_{k} \equiv 1$ near $K_{k}$ for $k \geq 1$. From Lemma 1 we see that for each $j \geq 1$, the function

$$
u_{j}(p) \equiv \frac{1}{\pi i} \int E(p, q) \varphi_{j}(q) \beta(q), \quad p \in \Omega,
$$

is smooth and satisfies $\partial \bar{\partial} u_{j}=\varphi_{j} \beta$. In particular, for each $k \geq 1$ we have

$$
\partial \bar{\partial}\left[u-\sum_{j=1}^{k} u_{j}\right]=\beta-\beta \sum_{j=1}^{k} \varphi_{j}=0 \quad \text { near } K_{k} \text {. }
$$

For each $k \geq 1$, the set $K_{k}$ has a fundamental system of open neighborhoods $S$ with the property that there are no compact connected components of $\Omega \backslash S$ $[\mathbf{7}, 3.10 .6]$; thus we may apply the Lax-Malgrange theorem $[\mathbf{7}, 3.10 .7]$ inductively to see that for each $k \geq 1$ there exists a harmonic function $w_{k}$ on $\Omega$ such that $\sup _{K_{k}}\left|u-\sum_{j=1}^{k}\left[u_{j}-w_{j}\right]\right|<1 / k$. We conclude that $u=\sum_{j=1}^{\infty}\left[u_{j}-w_{j}\right]$ on $\Omega$.

Now let $\varepsilon>0$ be arbitrary. Fix an integer $j \geq 1$. If $m_{j} \equiv \int\left|\varphi_{j} \beta\right|>0$, we conclude from Lemma 2 that for each point $q \in \operatorname{supp}\left(\varphi_{j} \beta\right)$ there exists a parametric disc $N_{q}$ such that $q \in N_{q} \subset \subset(\Omega \backslash F) \cap \Omega_{j}$ and

$$
|E(p, q)-E(p, s)| \leq \pi \varepsilon / m_{j} 2^{j+1} \quad \text { for } s \in N_{q}, p \in F \cup\left(\Omega \backslash \Omega_{j}\right) .
$$

Since $\operatorname{supp}\left(\varphi_{j} \beta\right)$ is compact, we can find a finite set $\mathscr{A}_{j} \subset \operatorname{supp}\left(\varphi_{j} \beta\right)$ such that $\operatorname{supp}\left(\varphi_{j} \beta\right) \subset \bigcup_{q \in \cup \cup_{j}} N_{q}$. Let $\left\{\eta_{q}\right\}_{q \in \mathscr{Q}_{j}}$ be a family of nonnegative functions in 
$C_{0}^{\infty}(\Omega)$ such that supp $\eta_{q} \subset N_{q}$ for each $q \in \mathscr{A}_{j}$ and $\sum_{q \in \mathscr{A}_{j}} \eta_{q} \equiv 1$ on a neighborhood of $\operatorname{supp}\left(\varphi_{j} \beta\right)$. We then define

$$
v_{j}(p)=\frac{1}{\pi i} \sum_{q \in \mathscr{A}_{j}} E(p, q) \int \eta_{q}(s) \varphi_{j}(s) \beta(s),
$$

and note that for each $p \in F \cup\left(\Omega \backslash \Omega_{j}\right)$ we have

$$
\begin{aligned}
\left|u_{j}(p)-v_{j}(p)\right|=\left|\frac{1}{\pi i} \sum_{q \in \mathscr{O}_{j}} \int[E(p, s)-E(p, q)] \eta_{q}(s) \varphi_{j}(s) \beta(s)\right| \\
\leq \frac{\varepsilon}{m_{j} 2^{j+1}} \int\left[\sum_{q \in \mathscr{O}_{j}} \eta_{q}(s)\right]\left|\varphi_{j}(s) \beta(s)\right|=\frac{\varepsilon}{m_{j} 2^{j+1}} \int\left|\varphi_{j} \beta\right|=\frac{\varepsilon}{2^{j+1}} .
\end{aligned}
$$

If $\int\left|\varphi_{j} \beta\right|=0$, then $u_{j} \equiv 0$; we then let $\mathscr{A}_{j}$ be the empty set and define $v_{j} \equiv 0$.

It follows that $h \equiv \sum_{j=1}^{\infty}\left[v_{j}-w_{j}\right]$ is well defined and finite at all points of $\Omega$ which are in the complement of the discrete set $\bigcup_{j=1}^{\infty} \mathscr{A}_{j}$. In fact, for each compact set $K \subset \Omega$ there is an index $J$ such that $v_{j}$ is (finite and) harmonic on $K$ for all $j \geq J$ and $\sum_{j=J}^{\infty}\left[v_{j}-w_{j}\right]$ is uniformly convergent on $K$; in particular, $\sum_{j=J}^{\infty}\left[v_{j}-w_{j}\right]$ is harmonic on int $K$. It follows that $h$ is essentially harmonic on $\Omega$. From (4) we also conclude that for each $p \in F, h(p)$ is finite and

$$
\begin{aligned}
|f(p)-h(p)| & =|u(p)-h(p)| \\
& =\left|\sum_{j=1}^{\infty}\left[u_{j}(p)-w_{j}(p)\right]-\left[v_{j}(p)-w_{j}(p)\right]\right| \\
& =\sum_{j=1}^{\infty}\left|u_{j}(p)-v_{j}(p)\right| \leq \sum_{j=1}^{\infty} \frac{\varepsilon}{2^{j+1}}=\frac{\varepsilon}{2}<\varepsilon .
\end{aligned}
$$

This completes the proof of the theorem.

\section{REFERENCES}

1. A. Boivin and P. M. Gauthier, Approximation harmonique sur les surfaces de Riemann, Canad. J. Math. 36 (1984), 1-8.

2. A. Dufresnoy, P. M. Gauthier, and W. H. Ow, Uniform approximation on closed sets by solutions of elliptic partial differential equations, Complex Variables 6 (1986), 235-247.

3. P. M. Gauthier, M. Goldstein, and W. H. Ow, Uniform approximation on unbounded sets by harmonic functions with logarithmic singularities, Trans. Amer. Math. Soc. 261 (1980), 169183.

4. P. M. Gauthier and W. Hengartner, Uniform approximation on closed sets by functions analytic on a Riemann surface, Approximation Theory (Z. Ciesielski and J. Musielak, eds.), Reidel, Dordrecht, 1975, pp. 63-70.

5. __ Approximation uniforme qualitative sur des ensembles non bornés, Les Presses de l'Université de Montréal, 1982.

6. M. Nakai and L. Sario, Classification theory of Riemann surfaces, Springer-Verlag, 1970.

7. R. Narasimhan, Analysis on real and complex manifolds, North-Holland, 1968. 47405

Department of Mathematics, Indiana University, Bloomington, Indiana 